\title{
End-to-End Delay Estimation using RPL Metrics in WSN
}

\author{
Pedro Pinto \\ ESTG, Instituto Politécnico de Viana \\ do Castelo and INESC TEC \\ Viana do Castelo and Porto, Portugal \\ Email: pedropinto@estg.ipvc.pt
}

\author{
António Pinto \\ CIICESI, ESTGF, Politécnico do \\ Porto and INESC TEC \\ Porto, Portugal \\ Email: apinto@inescporto.pt
}

\author{
Manuel Ricardo \\ INESC TEC, Faculdade de \\ Engenharia, Universidade do Porto \\ Porto, Portugal \\ Email: mricardo@inescporto.pt
}

\begin{abstract}
Critical monitoring applications can use wireless sensor networks to transport delay sensitive data. This data may demand bounded delays in order to be considered useful by the receiver. In these cases, an accurate and real-time estimation of the end-to-end delay could be used to anticipate the data usefulness prior to sending it.
\end{abstract}

A novel real-time and end-to-end delay estimation mechanism is proposed in this paper, which considers processing times and two new RPL metrics. Results show that our proposal is more accurate than the ETT-based solution for delay estimation, and it does not significantly degrade the network performance.

Keywords-WSN; QoS; End-to-End Delay; RPL; ETT; ETX

\section{INTRODUCTION}

Wireless Sensor Networks (WSN) can be used to transport data of monitoring applications. Critical monitoring applications can generate traffic flows with QoS requirements such as delay, loss, or throughput. If a particular flow is sensitive to delay, the WSN should deliver the packets of this flow to the destination within a delay that enables the data to be useful for the application.

From the communications point of view, a WSN node may generate data, forward data from other nodes, or consume data. A typical interaction between WSN nodes is presented in Fig. 1. For a flow generated in a given node, the End-to-End Delay (EED) can be defined as the time elapsed since data is generated at the application layer of the source node until this data arrives to the destination application in the sink node.
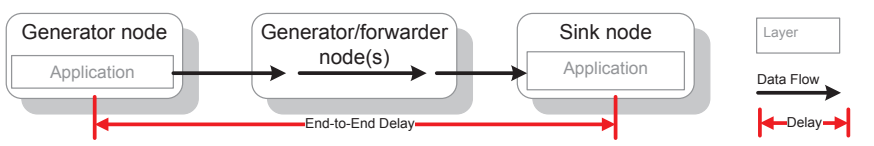

Fig. 1. WSN nodes and End-to-End Delay

In order to ensure an EED for a flow in a WSN scenario QoS mechanisms could be used. However, traditional QoS architectures such as integrated or differentiated services seem to be not suitable to WSN due to the additional functionality they would introduce in sensor nodes, such as traffic shaping, classification, policing, scheduling or resource reservation. Thus, a simpler but still accurate EED estimation mechanism needs to be defined. This mechanism could be applied to flows that are sensitive to delay but not to packet loss, and would be used to classify the data packets that will miss the application delay deadlines, and avoid their transmission to the network.

Current research efforts on quality of service characterize EED estimation by using probabilistic estimation of delays, network calculus, or routing metrics. Regarding routing metrics, the Expected Transmission Time (ETT) or ETT-based metrics have been proposed.

Our proposal consists of a novel EED estimation mechanism that combines path delays and node processing delays. Its novelty comes from considering in-node delays with two new RPL metrics. This solution provides an EED estimation which is more accurate than the ETT-based solution.

The structure of this paper is as follows. Section II surveys work related to our proposal. Section III describes the proposed EED estimation mechanism. Section IV presents the hardware and simulation environment used to validate our solution. Section V provides the results obtained and discusses them. Section VI concludes paper and addresses future work.

\section{RELATED WORK}

According to the taxonomy presented in [1], current efforts to measure or estimate EED can be divided in three types: queuing models or network calculus; active probing using messages and protocols; piggy-backing delay information into normal traffic or in routing protocol messages. In [2] is presented a probabilistic routing metric based on the estimation of the EED distribution. In [3] the authors developed a model to evaluate QoS by analyzing EED. In [4] network calculus was used to obtain the deterministic upper bound of EED in WSN. In [5] are used arrival and service curves for stochastic network calculus, used to derive the EED envelopes. The above proposals aim to obtain EED limits or bounds prior to WSN deployment, and do not obtain EED estimation in real time. The proposals using probe packets or piggy-back information in data packets have the undesired effect of introducing additional traffic in the WSN, contributing to consume energy and processing resources.

The research proposals using routing protocol messages are based on ETT or ETT-related metrics, which can either be obtained by using probe packets or by deriving it from metrics 
such as the Expected Transmission Count (ETX). According to [6], ETT can be derived from ETX by calculating:

$$
E T T=E T X \times \frac{S}{D}
$$

where ETX is the expected number of transmission attempts required for successfully transmitting a packet, $S$ is the packet size, and $D$ is the data rate of the link. A performance study regarding the use of ETX-based metrics can be found in [7]. In [8] a metric named Improved ETT is proposed, which focuses on the routing efficiency under various link conditions. In [9] the ETT metric is adapted to improve the estimation of transmission time by including the actual load of different nodes. In [10] the authors recommend that the queuing and transmission delay should be considered simultaneously in order to minimize EED. In [11] the authors present a novel ETT derived metric which takes into account the time between transmissions in each node in order to increase average network throughput in Wireless Mesh Networks. The research efforts presented above use ETT or ETT-based metrics to enhance performance, but the accuracy of these metrics was not discussed in any of these papers. Also, none of these proposals considers the processing delay which, in sensor nodes, may be relevant.

\section{END-TO-END DELAY ESTIMATION MECHANISM}

Let us assume 3 nodes: a generator, a forwarder and a sink. The forwarder node forwards packets from other nodes but also generates packets. The sink node is the destination of all packets. These nodes are represented in Fig. 2 where the generator node $i$ uses its parent node $p$, to reach the sink node $s$. Node $p$ also generates its own packets towards the sink. Fig. 2 also presents the layered communications architecture of these nodes, some of their relevant functions, and a data flow. The rounded-corner boxes inside each layer represent labels characterizing relevant states in the data communications process.

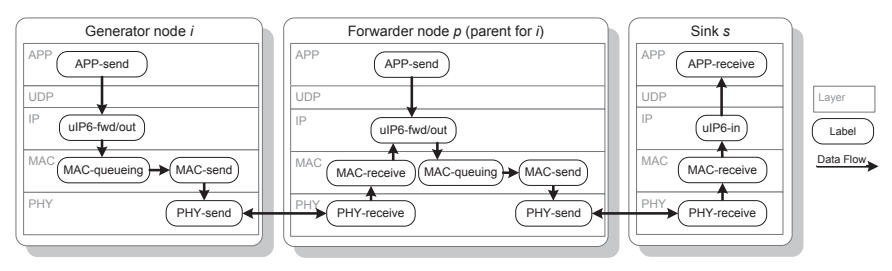

Fig. 2. EED estimation - WSN nodes' interaction

Our proposal estimates the EED by estimating all the delays between the labels where the data passes through, from the application in the generator node to the application in the sink node. The EED is estimated whenever a node's application generates a new data packet and it is based on the delays obtained for previous packets. Since our nodes run the ContikiOS 2.5 [12] operating system, the labels were inserted in the ContikiOS code files, according to Table I.

Within each node, two internal delay components are defined: the link delay, and the processing delay. The link delay comprises the time related to packet transmission and link queuing. The processing delay comprises the time elapsed while packets are being processed inside the nodes and that is not related to transmission or queuing. In order to obtain the delays in other nodes two RPL metrics are also used. So, in each node, the delay each packet will suffer is estimated from the delay of previous packets sent and from these two RPL metrics.

TABLE I. RELATION BETWEEN LABELS, FUNCTIONS AND FILES

\begin{tabular}{|c|c|c|}
\hline Label & Function in code & OS file \\
\hline APP-send & send_packet() & udp-client.c \\
\hline APP-receive & receive_packet() & udp-server.c \\
\hline uIP6-fwd/out & \multirow{2}{*}{ uip_process() } & \multirow{2}{*}{ uip6.c } \\
\hline uIP6-in & & \\
\hline MAC-receive & input_packet() & contikimac.c \\
\hline MAC-queuing & send_packet() & \multirow{4}{*}{ csma.c } \\
\hline MAC-send & \multirow{2}{*}{ transmit_queued_packet() } & \\
\hline PHY-send & & \\
\hline PHY-receive & mac_call_sent_callback() & \\
\hline
\end{tabular}

\section{A. Link Delay}

Fig. 3 shows the delays considered to obtain the link delay in node $i$, in particular the MAC layer queuing delay and the transmission delay. The link delay calculated for the packet $n$ transmitted from node $i$ to a parent node $p$ is obtained by:

$$
\text { LinkDelay }_{i p}^{n}=\text { QueueDelay }_{i}^{n}+\text { TransmissionDelay }_{i p}^{n}
$$

where the QueueDelay is the interval between the time the packet is inserted into the MAC queue until its removal, and the TransmissionDelay is the time interval required for the packet successful transmission, including the ACK reception.

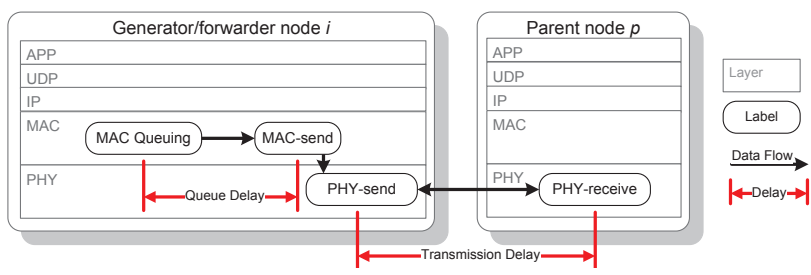

Fig. 3. Link Delay - Generator/forwarder node and parent node

\section{B. Processing Delay}

WSN nodes may have different capabilities in what concerns processing power and memory. In order to obtain the processing delay, different information paths inside a node are considered. In a generator node, the data payload will be generated by the application layer, then sent to the IP layer, and then passed to the MAC layer. In a forwarder node, after the packet is received, it is passed to the MAC layer, then to IP layer, and then it will be sent to the MAC queue. In the case of a sink node, the packet will be received, passed to the MAC layer, then to the IP layer, and finally delivered to the Application layer. These internal paths and associated delays are shown in Fig. 4. The delay names include the layers between which the delay is accounted (e.g. Delay_L5L3 is the delay between layer 5 and layer 3). Since the generation of data payload in layer 5 until the packet is received at the MAC layer, two internal delays are accounted: the Delay_L5L3 and the Delay_L3L2. For a generator node $i$, the Generation Processing Delay (GenProcDelay) for a packet $n$ is obtained as follows: 


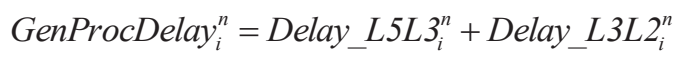

In the case node $i$ is forwarding a packet $n$ from other node, the following delays are accounted: Delay_L2L3_FWD which is the delay accounted between layer 2 and layer 3 of a packet meant to be forwarded - and Delay_L3L2. Thus, the Forward Processing Delay (FwdProcDelay) in node $i$ for packet $n$ is obtained by:

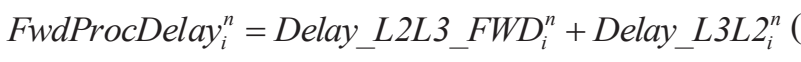

For the packet $n$ received in the sink $s$, Delay_L2L3 and Delay_L3L5 are accounted. The Input Processing Delay (InProcDelay) accounted in the sink is obtained by:

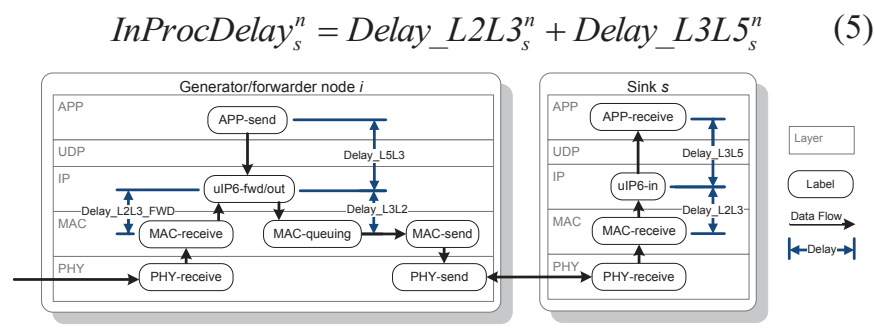

Fig. 4. Processing Delay - Generator/forwarder node and sink node

\section{Internal Delays}

The internal delays used in link delay and processing delay components are obtained by using timers with a millisecond precision. These timers measure the time interval between code execution points defined by the labels indicated in Table I. Exponential Weighted Moving Averages (EWMA) are used to consider the history of these timers:

$$
\text { Delay }_{i}^{n}=\beta \cdot \text { Delay }_{i}^{\text {last }}+(1-\beta) \cdot \text { Delay }_{i}^{n-1}
$$

These moving averages are used to obtain QueueDelay, TransmissionDelay, Delay_L2L3, Delay_L2L3_FWD Delay_L3L5, Delay_L5L3, and Delay_L3L2.

\section{RPL Metrics}

Our proposal uses the RPL (IPv6 Routing Protocol for Low-power and Lossy Networks)[13] as routing protocol. Nodes using RPL organize themselves in a tree-like topology named Destination-Oriented Directed Acyclic Graph (DODAG) optimized according to an Objective Function (OF) towards one defined sink node. Our proposal assumes RPL using the Minimum Rank with Hysteresis Objective Function (MRHOF)[14], which selects routes that minimize a metric using hysteresis in order to reduce instability due to small metric changes. The rank used by OF represents a cost for the path selected towards the root. By applying the OF, each node elects one parent towards the sink from a set of candidate parents. Different metrics may be used, carried in a DAG Metric Container object, which includes a set of Routing Metric/Constraint objects. In order to advertise the internal nodes delays, our proposal uses two metrics: the Path Delay Metric (PathDelayMetric) which represents the cumulative link delays to the sink, and the Processing Delay Metric (ProcDelayMetric) which represents the cumulative processing delays to the sink. Both metrics are advertised by every node within the RPL routing protocol messages. The
PathDelayMetric and ProcDelayMetric for a forwarding node $i$ with an RPL preferred parent $p$ and sink $s$, can be obtained by:

$$
\begin{gathered}
\text { PathDelayMetric }_{i s}=\text { LinkDelay }_{i p}^{n}+\text { PathDelayMetric }_{p s} \\
\text { ProcDelayMetric }_{i s}=\text { FwdProcDelay } \\
\end{gathered}
$$

A sink node $s$ advertises to its neighbors a PathDelayMetric of zero and a ProcDelayMetric according to the following:

$$
\text { ProcDelayMetric }_{s s}=\text { InProcDelay }{ }_{s}^{n}
$$

The other nodes will broadcast both path and processing metrics according to the RPL specification.

\section{E. In-node End-to-end Delay Estimation}

In our proposal, the EED estimation is composed of the end-to-end path delay which is the sum of all link delays, and the processing delay which is the sum of all processing delays. For a node $i$ with parent $p$, the Path Delay (PathDelay) of packet $n$ sent to the sink $s$ is calculated as follows:

$$
\text { PathDelay }_{i s}^{n}=\text { LinkDelay }_{i p}^{n}+\text { PathDelayMetric }_{p s}
$$

The PathDelay is obtained by the same elements of the PathDelayMetric (Eq. 6). However, it is calculated when a packet is generated while the PathDelayMetric is calculated when RPL advertisements need to be sent to the neighbors, which in turn depends on the hysteresis function.

For a packet $n$ generated by node $i$ and sent to the sink $s$, the Processing Delay (ProcDelay) estimated in node $i$ is the following:

$$
\text { ProcDelay }_{i s}^{n}=\text { GenProcDelay }_{i}{ }^{2} \text { ProcDelayMetric }_{p s}
$$

The EED estimation mechanism is presented in Fig. 5 and it is implemented in all nodes of the WSN.

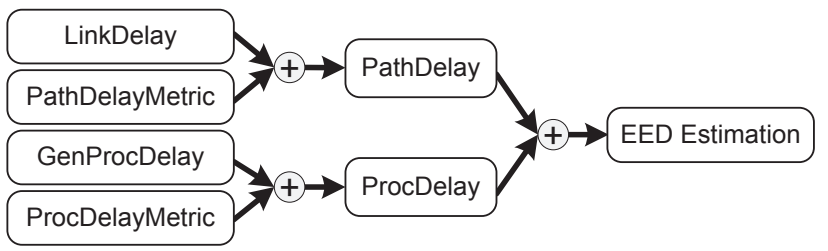

Fig. 5. EED Estimation using PathDelay and ProcDelay

When a node $i$ needs to send a data packet $n$ it estimates the EED towards sink $s$ using PathDelay and the ProcDelay obtained for the last packet as follows:

$$
\text { EstimatedEED } D_{i s}^{n}=\text { PathDelay }_{i s}^{n-1}+\text { ProcDelay }_{i s}^{n-1}
$$

\section{HARDWARE AND SimUlation ENVIRONMENT}

A test scenario was deployed and the Contiki Cooja Simulator [15] was used to validate our EED estimation proposal. The network topology adopted is shown in Fig. 6 and the simulation parameters are presented in Table II. The simulated scenario consists of 16 generator/forwarder nodes placed within a distance of 30 meters from each other plus a sink node, deployed in a WSN area of 100 meters by 100 meters. Each node was simulated as a Tmote Sky[16] composed of a MSP430F1611 micro-controller and a CC2420 
radio with a data rate of $250 \mathrm{kbit} / \mathrm{s}$ using IEEE 802.15.4 MAC and PHY layer specifications, with transmission and interference ranges of 30 meters, and using the Unit Disk Graph Medium as physical channel model. The nodes run the Contiki OS 2.5[12] and were programmed to enable the debug of application and RPL messages. Extra code was programmed to implement the timers in each node and the respective processing delay was measured, having an impact of $16 \mathrm{~ms}$ per processed packet. The application layer uses UDP as transport layer and it generates packets of 100 Bytes in a Constant Bit Rate (CBR) by using constant Inter-packet Generation Intervals (IGIs). The simulations were repeated 10 times using different seeds. Simulations were configured to stop when the sink has received 500 packets from each node.

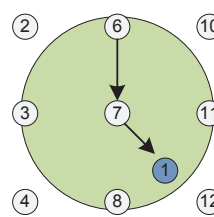

(14)
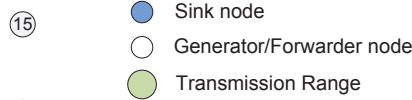

$\downarrow$ Data Flow

(5) (13) (17)

Fig. 6. Simulation Topology

TABLE II. SimUlation PARAMETERS

\begin{tabular}{|c|c|}
\hline Parameter & Value \\
\hline Number of nodes & $16+$ sink node \\
\hline Deployment area & $100 \mathrm{~m} \times 100 \mathrm{~m}$ \\
\hline Transmission range & $30 \mathrm{~m}$ \\
\hline Channel model & Unit Disk Graph Medium \\
\hline Packet size & 100 Bytes \\
\hline Transport/Application & UDP/CBR \\
\hline
\end{tabular}

In the simulation, the ETT-based solution was compared against our proposal and the EED estimation is obtained using the Eq. 1. RPL was configured to use the ETX metric. The metric value is multiplied by a transmission ratio in order to obtain the estimation of the EED as shown in Fig. 7.

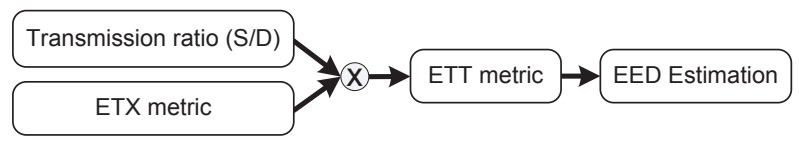

Fig. 7. EED estimation using ETT-based solution

The simulator was configured to output the time instant when a packet is generated and when a packet reaches the destination application. In order to caracterize the EED estimation accuracy, when a packet is generated an EED estimation is performed, saved, and compared later with the real EED. The absolute value of the difference between the estimation and the real results is taken as the EED estimation error. During simulations the EED estimation errors for all packets and for all the nodes are accounted. When simulation ends the average of these errors is calculated as the average EED estimation error. Confidence intervals of $90 \%$ are obtained.

\section{RESULTS AND ANALYSIS}

Fig. 8 shows the average EED estimation error and respective confidence intervals for both solutions, for IGIs ranging from 1 to 10 seconds in steps of 1 second. The results show that, for IGIs below 2 seconds the average estimation error of our proposal is higher than the obtained for the ETTbased solution. For an IGI equal or larger than 2 seconds (smaller traffic loads), our proposal presents an average estimation error below the ETT-based solution. For an IGI higher than 3 seconds, the difference obtained from the both solutions is approximately constant, having a value around 250 ms.

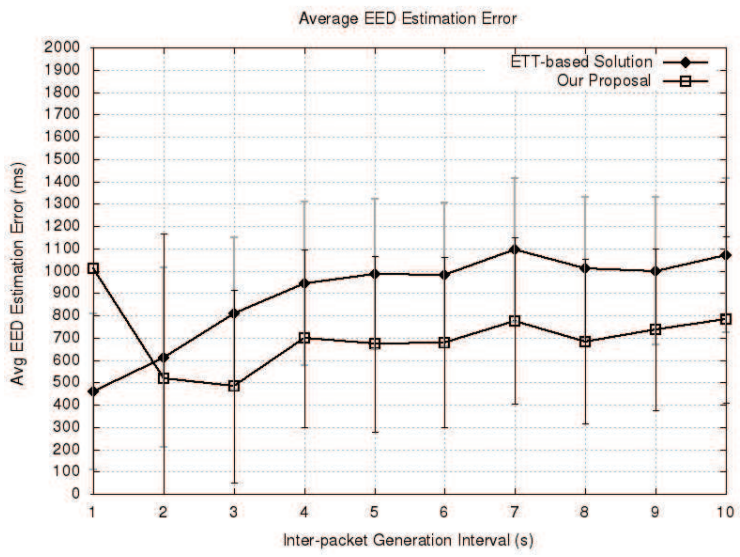

Fig. 8. Average EED Estimation Error - IGI from 1 to $10 \mathrm{~s}$

Fig. 9 zooms Fig. 8 and shows the average EED estimation error for both solutions obtained for IGI but ranging from 0.5 to 5 seconds, in steps of 0.5 seconds. The results show that, for IGIs shorter than 1.5 seconds, our proposal presents an higher estimation error than the ETT-based solution. For an IGI value of 1.5 seconds the average estimation error obtained for both solutions is approximately the same; above that value, our proposal presents lower average estimation errors.

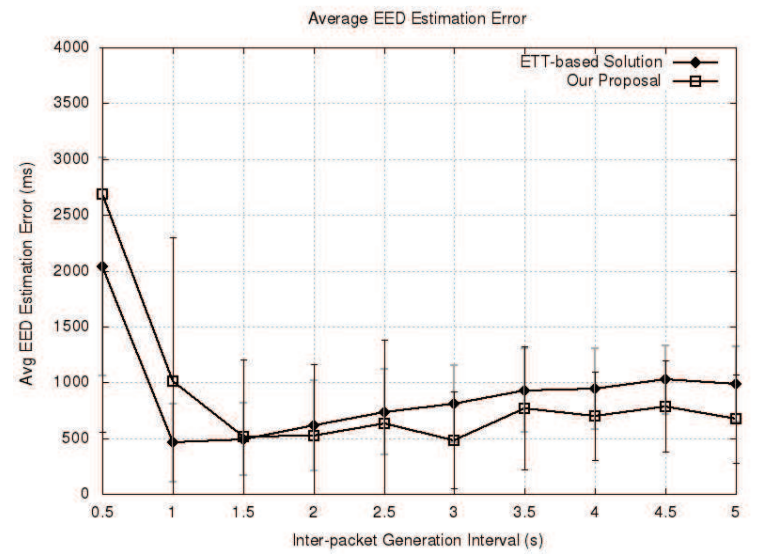

Fig. 9. Average EED Estimation Error - IGI from 0.5 to $5 \mathrm{~s}$

Fig. 10 shows the average PathDelay and ProcDelay distributions of the EED estimation for our proposal and IGIs ranging from 0.5 to 5 seconds. For IGIs ranging from 0.5 to 2.5 seconds the ProcDelay component accounts 5\% of the total EED estimation and it increases gradually up to $35 \%$. For IGIs larger than 2.5 seconds the ProcDelay component becomes 
approximately constant and it accounts for about $35 \%$ of the EED estimation. From the results shown in Fig. 9 and Fig. 10 we conclude that, for IGIs below 1.5 seconds, the PathDelay has an impact higher than the ProcDelay. For these high network loads, the PathDelay suffers from the links instability and it turns highly unpredictable making our estimation less accurate. For IGIs above 1.5 seconds, the ProcDelay represents around $30 \%$ of the EED estimation and our proposal presents higher accuracy than the obtained by the ETT-based solution, benefiting from the consideration of processing delays.

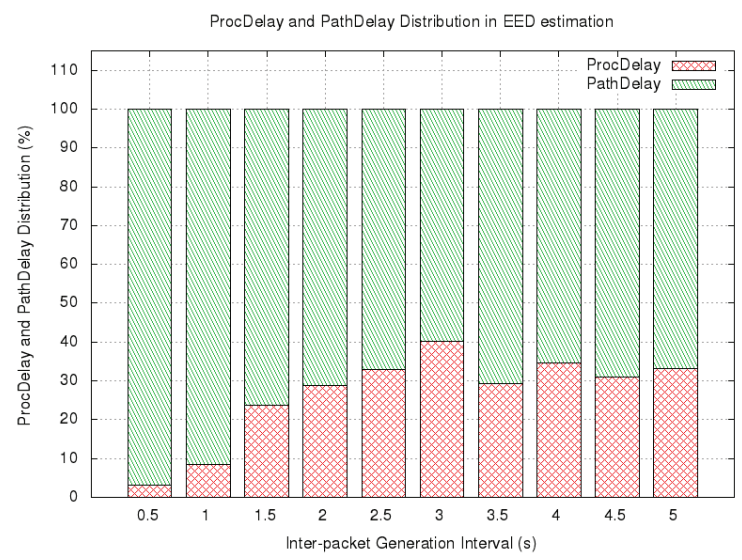

Fig. 10. ProcDelay and PathDelay Distribution - IGI from 0.5 to $5 \mathrm{~s}$

Fig. 11 presents the average number of RPL packets sent by both solutions, per node and per simulation, for IGIs ranging from 0.5 to 5 seconds. The results show that, for all IGIs, the number of RPL packets generated by our proposal is 3 times the number of packets generated by the ETT-based solution. From the results shown in Fig. 9 and Fig. 11 we can conclude that the new RPL metrics used in our proposal lead to an higher advertisement rate due to the ProcDelayMetric and PathDelayMetric changes that occur more often than the ETX metric. Thus, for shorter IGIs (high loads), our proposal has higher estimation errors than the ETT-based solution. This high advertisement rate becomes a benefit for our proposal for IGIs larger than 1.5 seconds, since it enables a more accurate estimation.

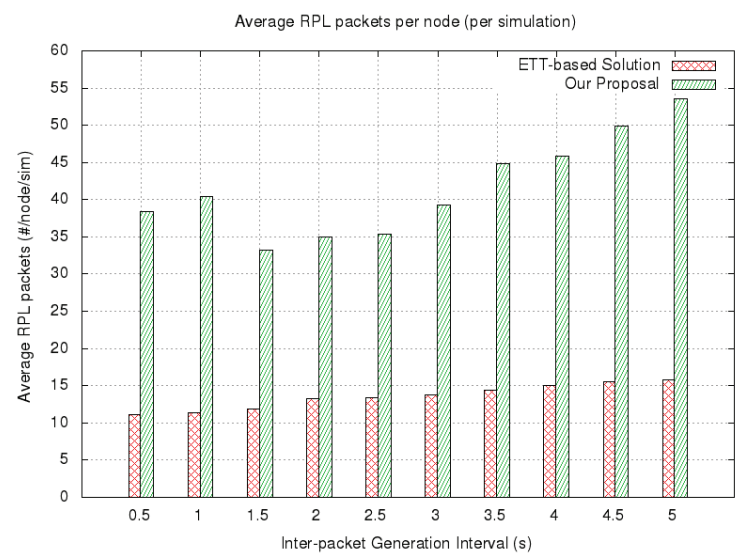

Fig. 11. Average number of RPL packets - IGI from 0.5 to $5 \mathrm{~s}$

Fig. 12 and Fig. 13 present the average EED estimation error for both solutions obtained for specific nodes (node 8 and node 5), for IGIs ranging from 1 to 10 seconds. Fig. 12 presents the results for node 8 which has the sink as parent. Fig. 13 presents the results for the node 5 which has two forwarder nodes in the path for the sink. The results show that nodes closer to the sink (e.g. node 8) have estimation errors smaller than the nodes more distant from the sink (e.g. node 5). Also, for the nodes far from the sink, the difference between the estimations using ETT-based solution and our proposal is higher. For IGIs shorter than 6 seconds, the estimation in nodes far from the sink becomes largely inaccurate, when compared to nodes closer to sink. For IGI values of 1 and 2 seconds in node 5 , results show that there were no packets arriving destination and so the average estimation error is zero.

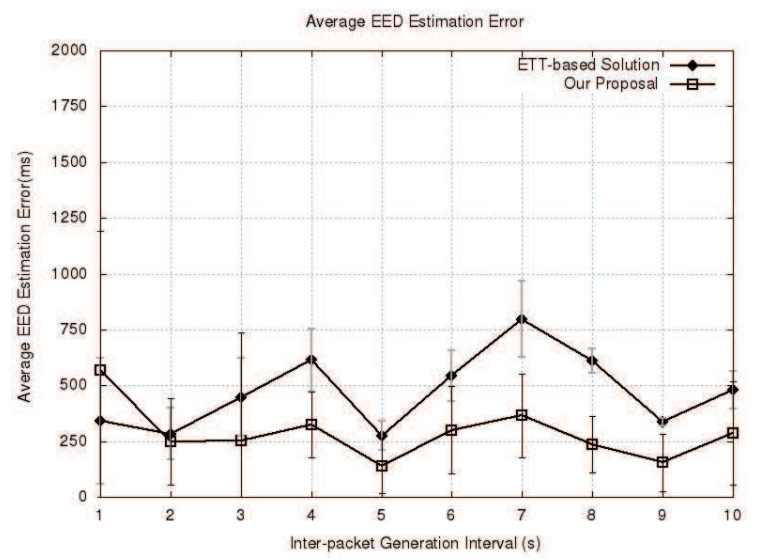

Fig. 12. Average EED Estimation Error - node 8

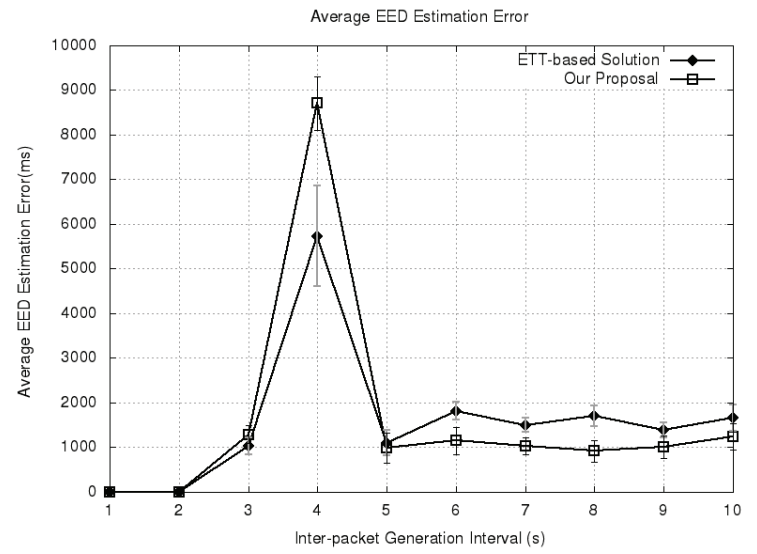

Fig. 13. Average EED Estimation Error - node 5

Fig. 14 presents the average EED for both solutions and for IGIs ranging from 1 to 10 seconds. The results show that, for almost all IGIs, the average EED obtained for our proposal is higher than the obtained for ETT-based solutions, except for the IGI of 3 seconds. At the same time, for IGIs shorter than 3 seconds, the difference of both solutions is accentuated. The results in Fig. 14 also show that the average EED values are closer to the estimate EED errors presented in Fig. 8. Our proposal intends to take advantage of the maximum EED verified and, for an IGI of 5 seconds, it presents an average EED of $1000 \mathrm{~ms}$ and an error around $690 \mathrm{~ms}$. Thus, in this case it is expected that a packet reachs the sink at maximum of 1690 ms for our proposal against around $2000 \mathrm{~ms}$ for the ETT-based proposal. 


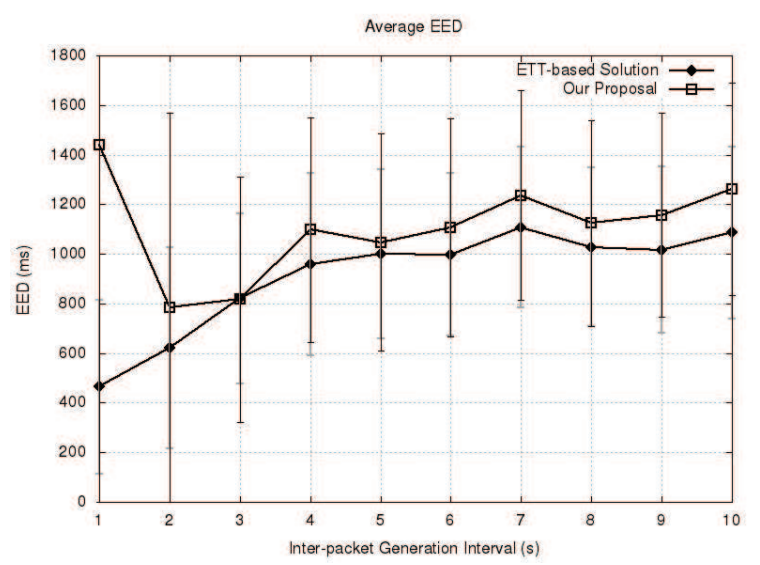

Fig. 14. Average EED

Fig. 15 presents the Packet Reception Ratio (PRR) for both solutions and for IGIs ranging from 1 to 10 seconds. The results show that for IGIs shorter than 7 seconds, the PRR of our proposal is less than the PRR obtained for the ETT-based solution, with a constant difference of approximately $10 \%$. The results presented in Fig. 14 and Fig. 15 indicate that the proposed mechanism has no significant impact on these performance items for an IGI higher than 7 seconds. For IGI shorter than 3 seconds, the average EED increases significantly when compared to ETT-based solution; for an IGI shorter than 7 seconds, the PRR is affected in approximately $10 \%$. This impact is due to the higher refresh rates of the RPL metrics used in our proposal, as explained above.

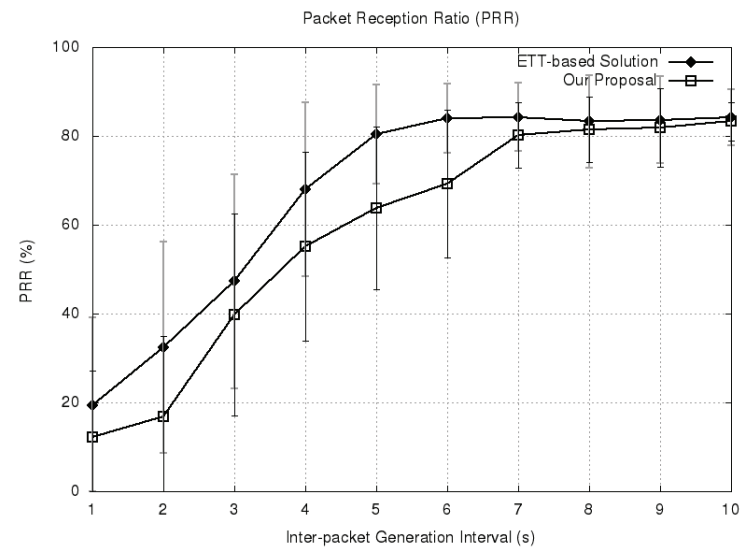

Fig. 15. Packet Reception Ratio

\section{CONCLUSIONS AND FUTURE WORK}

In this paper is proposed a novel real-time mechanism used to estimate per-packet end-to-end delay for monitoring applications running in WSN. This proposal accounts both the transmission and processing delays of previous packets to estimate the EED for each new packet. The EED estimation is obtained by combining internal timers with two cumulative RPL metrics.

Our proposal was compared against an ETT-based solution and the results show that our proposal produces a more accurate EED estimation for low network loads, without impacting significantly on the network performance in terms of average EED and PRR values.

Our proposal can be used to provide a node with EED information before it transmits a packet. By dropping useless packets a network can see its performance improved. These mechanisms can also be used to save nodes energy by avoiding the transmission of useless data.

\section{ACKNOWLEDGMENT}

This work is partially financed by the ERDF - European Regional Development Fund through the COMPETE Programme and by National Funds through the FCT Fundação para a Ciência e a Tecnologia within project CMUPT/SIA/0005/2009.

\section{REFERENCES}

[1] R. Baumann, S. Heimlicher, M. Strasser and A. Weibel and S. H. R. Baumann, "A survey on routing metrics," TIK Rep. 262 ETH-Zent., 2007.

[2] R. S. Oliver and G. Fohler, "Probabilistic estimation of end-to-end path latency in Wireless Sensor Networks," in IEEE 6th International Conference on Mobile Adhoc and Sensor Systems, 2009. MASS '09, 2009, pp. 423-431.

[3] D. D. Chaudhary and L. M. Waghmare, "Quality of service analysis in wireless sensor network by controlling end-to-end delay," in 2012 7th IEEE Conference on Industrial Electronics and Applications (ICIEA), 2012, pp. 703-708.

[4] L. Cui and J. Yong-feng, "The research on end-to-end delay upper bound in wireless sensor network," in 2012 International Conference on Image Analysis and Signal Processing (IASP), 2012, pp. 1-4.

[5] B. Huhu and G. Wang, "Study on Delay Boundary Based on SNC in Wireless Sensor Networks," in 2011 7th International Conference on Wireless Communications, Networking and Mobile Computing (WiCOM), 2011, pp. 14.

[6] R. Draves, J. Padhye, and B. Zill, "Routing in multi-radio, multi-hop wireless mesh networks," in In ACM MobiCom, 2004, pp. 114-128.

[7] N. Javaid, A. Javaid, I. A. Khan, and K. Djouani, "Performance study of ETX based wireless routing metrics," in Computer, Control and Communication, 2009. IC4 2009. 2nd International Conference on, 2009, pp. $1-7$.

[8] S. Biaz, B. Qi, and Y. Ji, "Improving Expected Transmission Time Metric in Multi-Rate Multi-Hop Networks," in Consumer Communications and Networking Conference, 2008. CCNC 2008. 5th IEEE, 2008, pp. $533-$ 537.

[9] H. Zhou, C. Huang, Y. Cheng, and G. Wang, "A New Multi-metric QoS Routing Protocol in Wireless Mesh Network," in Networks Security, Wireless Communications and Trusted Computing, 2009. NSWCTC '09. International Conference on, 2009, vol. 1, pp. $459-467$.

[10] H. Li, Y. cheng, C. Zhou, and W. Zhuang, "Minimizing End-to-End Delay: A Novel Routing Metric for Multi-Radio Wireless Mesh Networks," in IEEE INFOCOM 2009, 2009, pp. $46-54$.

[11] D. Teng, S. Yang, D. Wang, and Y. Hu, "NQETT: Node Quality Adjusted ETT for Wireless Mesh Networks," in Wireless Communications, Networking and Mobile Computing, 2008. WiCOM '08. 4th International Conference on, 2008, pp. $1-4$.

[12] "Contiki OS." [Online]. Available: http://www.contiki-os.org.

[13] T. W. <wintert@acm.org>, "RPL: IPv6 Routing Protocol for LowPower and Lossy Networks." [Online]. Available: http://tools.ietf.org/html/rfc6550.

[14] O. G. <gnawali@cs.uh.edu>, “The Minimum Rank with Hysteresis Objective Function.” [Online]. Available: http://tools.ietf.org/html/rfc6719.

[15] F. Osterlind, A. Dunkels, J. Eriksson, N. Finne, and T. Voigt, "CrossLevel Sensor Network Simulation with COOJA," in Proceedings 200631 st IEEE Conference on Local Computer Networks, 2006, pp. 641-648.

[16] "Tmote Sky Project." [Online]. Available: http://www.snm.ethz.ch/Projects/TmoteSky. 